\title{
Omega-3 fatty acids and brain energy metabolism: Impact on the expression of glucose transporters and glucose transport activity in endothelial cells in culture
}

F. Pifferi ${ }^{1}$

M. Jouin ${ }^{1}$

F. Roux ${ }^{2}$

N. Perrière ${ }^{2}$

J-M. Alessandri ${ }^{1}$

M. Lavialle ${ }^{1}$

P. Guesnet ${ }^{1}$

1. Nutrition et régulation lipidique des fonctions cérébrales (Nu.Re.Li.Ce), UR909, INRA F-Jouy-en-Josas, INRA, Jouy-en-Josas

2. INSERM U705, CNRS UMR 7157, Hôpital Fernand Widal, F-Paris

Alterations in cognitive performance and neuronal activity in animals deficient in $\mathrm{n}-3$ polyunsaturated fatty acids (PUFA) have been linked to the decrease in docosahexaenoic acid (22:6n-3, DHA) level in brain membranes. These alterations could partly result from changes in brain energy metabolism and namely in glucose transport activity. Indeed, we reported that several brain areas of rats raised on a diet deficient in n-3 PUFAs have below-normal rates of glucose utilization [1]. We postulated that the quantity of glucose transporter GLUT1 in deficient rats was functionally abnormal as its immunoreactivity was low. We present experimental data that evaluated: 1) the in vivo impact of an n-3 PUFA-deficient diet on expression of the main glucose transporters of rat cerebral cortex (endothelial 55-kDa GLUT1, astrocytic 45-kDa GLUT1, and neuronal GLUT3); and 2) the in vitro impact of the three main long-chain n-6 and n-3 PUFAs, arachidonic acid (20:4n-6), eicosapentaenoic acid (20:5n-3) and DHA, on the fatty acid composition of membrane phospholipids and glucose uptake in two models of rat brain endothelial cells (primary cultures (RBEC), and immortalized cell line RBE4).

In vivo study - Rats raised with a n-3 PUFA deficient diet throughout one generation were compared with rats fed a diet with adequate amounts of $n-6$ and n-3 PUFA. The fatty acid profile of brain microvessels was determined in order to identify the alterations in n-6 and n-3 PUFA in endothelial cells compared to their profile in neural cells from the cerebral cortex. The main glucose transporters were quantified by western immunoblotting and by real time quantitative RT-PCR. Specific binding of cytochalasin B to GLUT1 of isolated microvessels was also determined.

In vitro study - The three PUFA studied were added to the culture medium $(15 \mu \mathrm{M})$ as sodium salts on the first day of culture and until the cells were used. Glucose uptake was measured on monolayers of RBEC and RBE4 cells using the non-metabolisable radiolabelled glucose analogue 3-o-methylglucose ([$\left.\left.{ }^{3} \mathrm{H}\right]-3-0-M G\right)$.

In vivo data showed that the 55-kDa GLUT1 protein quantity decreased by $25 \%$ in the n-3 PUFA-deficient microvessels, as did $45 \mathrm{kDa}-\mathrm{GLUT1}$ in the homogenate (30\% decrease). Results from cytochalasin B binding confirmed these data, showing a lower level of its maximum binding to GLUT1. However, GLUT3 protein quantity did not change as GLUT1 and GLUT3 mRNA level. In vitro data demonstrated that both cerebral endothelial cells had low n-6 (mainly 20:4n-6) and n-3 (mainly 22:6n-3) PUFA concentrations under standard conditions of culture. RBEC and RBE4 cells grown in medium supplemented with 20:4n-6, $20: 5 n-3$ or $22: 6 n-3$ ( $15 \mu \mathrm{M}$, physiological concentrations), avidly incorporated these PUFAs into their membrane phospholipids to approach the contents of cerebral microvessels of control rats (considered as physiological references). However RBE4 cells did not incorporate sufficient 20:4n-6 compared to physiological concentrations. Supplementation with $20: 5 n-3$ and $22: 6 n-3$ increased the basal glucose transport in RBEC $\left(\left[{ }^{3} \mathrm{H}\right]-3-\mathrm{O}-\right.$ methylglucose uptake) by $50 \%$ and $35 \%$ respectively, while $20: 4 n-6$ had no effect. On the other hand, none of the PUFA supplements had a significant effect on glucose uptake by RBE4. We demonstrated that incorporation of $n-3$ long-chain PUFAs in RBEC membranes acts on glucose transport activity.

We suggest that the decreased glucose utilization in the cerebral cortex of n-3 PUFA-deficient rats is in part due to reduced amounts of the two GLUT1 isoforms, possibly issuing from post-transcriptional down-regulation of GLUT1 protein synthesis. In vitro studies suggest that n-3 PUFA can directly modulate the activity of glucose transport in endothelial cells by altering their membrane phospholipid content.

\section{Reference}

1. Ximenes et al., 2002.

POSTERS 